\title{
Unruptured right coronary sinus to left ventricle aneurysm diagnosed by cross sectional echocardiography
}

\author{
C K MOK, K L CHEUNG, R Y C WANG \\ From the Departments of Surgery and Medicine, University of Hong Kong, Grantham Hospital, Hong Kong
}

SUMMARY A 37 year old Chinese man presenting with features of chest infection was diagnosed as having severe aortic regurgitation. Cross sectional echocardiography showed an intact right coronary sinus of Valsalva aneurysm, which protruded into the left ventricle. The changes in appearance of the aneurysm during the cardiac cycle were recorded by cross sectional echocardiography. The opening of the aneurysm was successfully closed and the aortic valve replaced with a mechanical prosthesis.

Although uncommon, a sinus of Valsalva aneurysm is a well known clinical entity. Most aneurysms of the sinus of Valsalva originate in the right coronary sinus and penetrate into either the right ventricle or atrium. ${ }^{12}$ An aneurysm of the right coronary sinus which extends into the interventricular septum and protrudes into the left instead of the right ventricle is exceedingly rare. To our knowledge, only a few cases have been reported. ${ }^{3}$ Of the patients reported, all had ruptured aneurysms except one. ${ }^{3}$ Recently, we encountered a patient with severe aortic regurgitation secondary to an unruptured right coronary sinus aneurysm, which extended into the left ventricular outflow tract and protruded against the ostium of the left ventricle. The aortic sinus aneurysm was diagnosed by cross sectional echocardiography and subsequently confirmed by angiography and at operation. Because of the rarity of the anatomical anomaly and because the cross sectional echocardiographic features of such an aneurysm have not been described before, we report this case.

\section{Case report}

A 37 year old Chinese man was admitted to hospital in December 1983 with progressive exercise intolerance during the past two months and a cough of two weeks'

Requests for reprints to Professor C K Mok, Division of Cardiothoracic Surgery, Department of Surgery, University of Hong Kong, Grantham Hospital, 125 Wong Chuk Hang Road, Hong Kong. duration. The cough produced yellowish sputum, which was occasionally blood streaked. He was afebrile, and there was no history of notable past illnesses. At physical examination on admission his general condition was satisfactory. There was no evidence of congestive heart failure. His blood pressure was 140/ $50 \mathrm{~mm} \mathrm{Hg}$ and his pulse 90 beats/min, regular, and collapsing in character. The heart was enlarged with a left ventricular impulse, and the apex beat was in the sixth intercostal space $2 \mathrm{~cm}$ lateral to the midclavicular line. A 3/6 ejection systolic and an early diastolic murmur were heard along the left sternal border. Coarse inspiratory and expiratory crackles were present over the right lung base. Laboratory data showed no abnormalities. The electrocardiogram showed sinus tachycardia and features of left ventricular hypertrophy. A chest radiograph showed cardiomegaly (cardiothoracic ratio 0.61 ) and diffuse mottling over the right lung.

Echocardiography indicated a grossly dilated left ventricle (left ventricular end diastolic dimension 7.5 $\mathrm{cm}$ and end systolic dimension $5.5 \mathrm{~cm}$ ). The aortic root was $3 \mathrm{~cm}$ in diameter, and the aortic valve had three cusps. A $2 \mathrm{~cm}$ lobulated pulsating mass dominated the subaortic area and was readily seen in the parasternal long axis and short axis views as well as in the apical four chamber view (Fig. 1). The mass extended from the region of the right coronary cusp to the adjoining interventricular septum. In the parasternal long axis view it enlarged during diastole and descended into the left ventricle during diastole and moved towards the anterior leaflet of the opened 


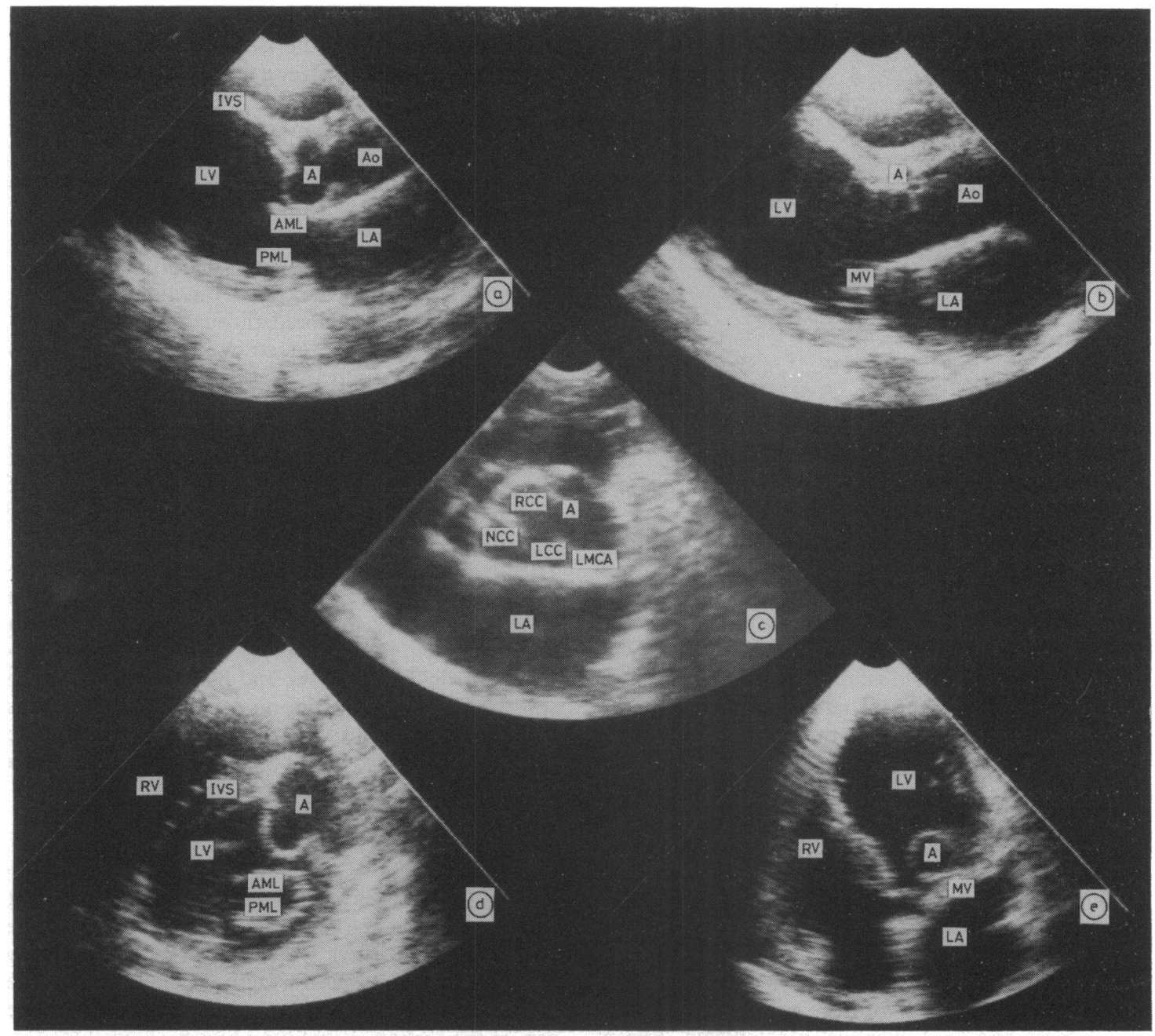

Fig. 1 Cross sectional echocardiograms of the unruptured right coronary sinus of Valsalva aneurysm in (a) the parasternal long axis view at end diastole; (b) the parasternal long axis view at end systole; (c) and (d) the parasternal short axis views and (e) the apical four chamber view. $A$, aneurysm; $A$ o, aorta; $L A$, left atrium; $L V$, left ventricle; $R V$, right ventricle; $I V S$, interventricular septum; $M V$, mitral valve; $A M L, P M L$, anterior and posterior mitral leaflets respectively; LMCA, left main coronary artery.

mitral valve. At end diastole it almost filled the left ventricular outflow tract (Fig. 1a). In systole it contracted down and almost disappeared at end systole (Fig. 1b). The mitral valve moved normally, and diastolic fluttering of the anterior leaflet was evident. The cross sectional echocardiographic features were compatible with severe aortic regurgitation, and we thought that the pulsating mass could be an unruptured aneurysm of the right coronary sinus of Valsalva.

In view of the clinical and radiographic features of concomitant chest infection, the patient was treated with erythromycin after sputum samples were taken for bacterial, including mycobacteria, and viral culture. Nevertheless, all cultures yielded no growth, and there was no improvement in his chest radiograph after four weeks. Bronchoscopy with a fibreoptic bronchoscope did not show any endobronchial lesion. Cultures of bronchial aspirates were also negative. A transbronchial biopsy specimen showed features of organising pneumonia. He was then given a course of intravenous tobramycin and cefotaxime empirically. The radiographic mottling in the right lung field gradually resolved over the following three weeks. 


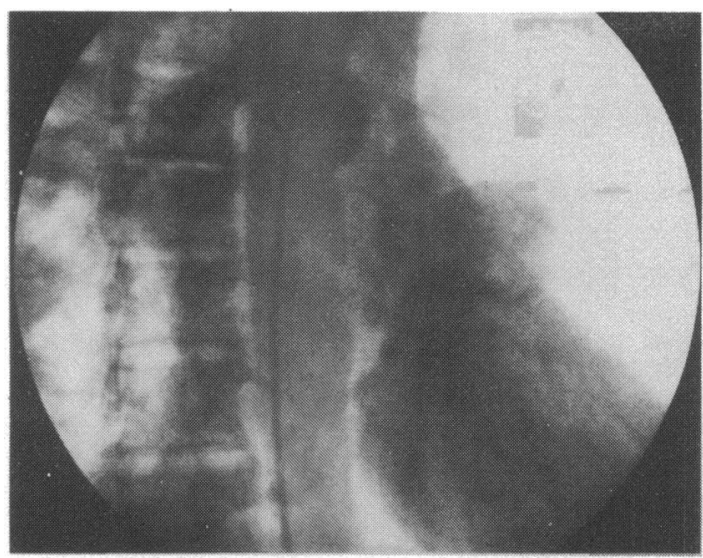

Fig. 2 Angiogram of aortic root in the right anterior oblique view. There is filling of the aneurysm and aortic regurgitation.

While awaiting further investigation and cardiac surgery he developed acute left heart failure and pulmonary oedema. This was successfully controlled with a diuretic and a vasodilator. Repeated echocardiographic examination showed similar features with further left ventricular dilatation and development of premature closure of the mitral valve. Immediate cardiac catheterisation showed a mean pulmonary artery pressure of $40 \mathrm{~mm} \mathrm{Hg}$, a mean pulmonary artery wedge pressure of $34 \mathrm{~mm} \mathrm{Hg}$, and a left ventricular end diastolic pressure of $24 \mathrm{~mm} \mathrm{Hg}$. The cardiac index was $1.44 \mathrm{l} / \mathrm{min}$ per $\mathrm{m}^{2}$. No pressure gradient nor intracardiac shunt were found. A cineangiogram (Fig. 2) confirmed severe aortic regurgitation and the presence of an unruptured aortic sinus aneurysm.

\section{OPERATIVE FINDINGS AND REPAIR}

Surgery was performed with the aid of cardiopulmonary bypass and cold chemical cardioplegia. Except for the severe left ventricular hypertrophy, the heart and great vessels were normal externally. At aortotomy the aortic valve consisted of three cusps, the left and non-coronary cusps being slightly thickened and near normal size. The right coronary cusp was small, fibrotic, and centrally displaced. This small deformed cusp was probably the cause of the aortic regurgitation. A $15 \times 10 \mathrm{~mm}$ opening was present in the left and central parts of the right coronary sinus of Valsalva. It was bounded posteromedially by the displaced right coronary cusp and anterolaterally by the aortic wall. It appeared as if the aortic valve annulus at the right coronary sinus had been detached from the aortic wall. This opening led to a lobulated, smooth surface cul de sac in the interventricular septum. When this sac was filled with fluid it bulged into the left ventricle towards the anterior leaflet of the mitral valve. The coronary ostia were normal, and there was no other abnormality at the aortic root. An attempt was made to reattach the displaced right coronary cusp to the aortic wall. Nevertheless, this further distorted the aortic valve, which was then excised. The remnant of the right coronary cusp was sutured to the aortic wall by several horizontal mattress sutures buttressed with teflon felt pledgets. This removed the aneurysm completely from the aortic root. A 25A Medtronic Hall valve was then sutured in the subcoronary position. The heart was defibrillated without difficulty and resumed normal function. The right pleural cavity was explored through the sternotomy incision, and one litre of straw coloured fluid was sucked out. The right lung appeared to be oedematous but otherwise normal. The patient was returned to the intensive care ward in a satisfactory condition.

Thirty six hours after the operation, he developed recurrent ventricular tachycardia and episodes of transient complete heart block. Each episode of arrhythmia was accompanied by low cardiac output. The arrhythmia was finally controlled with intravenous amiodarone and temporary atrial pacing. Acute renal failure developed, which was treated with haemodialysis. He gradually recovered and was discharged from hospital one and half months after the operation. At follow up one month later he was asymptomatic, and repeated echocardiographic examination showed only a dilated left ventricle with a normal functioning aortic prosthetic valve.

\section{HISTOPATHOLOGY}

Histopathological examination of the excised aortic valve cusps showed patchy hyalinisation and areas of myxomatous change particularly of the fibrosa. The appearance suggested a degenerative lesion. Biopsy of the aortic wall showed hyalinised thickening of the intima, and the media appeared to be deficient in one part of the section. Right lung biopsy showed moderate congestion, patchy fibrosis particularly of interlobular regions, and patchy thickening of interalveolar septa with mild sclerosis of small vessels. Subpleural fibrosis with infiltration by lymphocytes, plasma cells, and some macrophages was also present. The features were those of moderate passive congestion with patchy fibrosis.

\section{Discussion}

Aneurysm of sinus of Valsalva is a morphological diagnosis and includes both congenital and acquired aneurysms. Acquired aneurysms are mainly due to syphilis, tuberculosis, or infective endocarditis. As pointed out by Sakakibara and Konno, ${ }^{2}$ the site of origin and direction of dilatation of congenital aortic sinus aneurysms follow a definite pattern. They are determined by the developmental process in the 
embryonic period. Over $95 \%$ of aneurysms originate in the right coronary sinus and right part of the noncoronary sinus and protrude into the right ventricle or atrium. On the other hand, acquired aneurysms affect tissues at random and protrude or rupture in directions that are inexplicable by embryology. In our patient, there was no clinical or pathological evidence of syphilis, tuberculosis, or infective endocarditis. His right coronary sinus aneurysm probably belonged to the classification of pseudoaneurysms as described by Sakakibara and Konno. ${ }^{2}$ These aneurysms develop after intramural rupture of a congenital aneurysm with subsequent formation of a haematoma and organisation. What initiated the intramural rupture in our patient is unexplained.

The extension of an unruptured aortic sinus aneurysm into the interventricular septum and thence into the left ventricle is extremely rare. Only one case has been reported by Metras and associates. ${ }^{3}$ In their report they cited seven other cases seen by French workers, which are not available to us. They stated that the French workers reported that septal extension of an aortic sinus aneurysm might present as mitral regurgitation because of displacement of the anterior mitral leaflet. In our patient, however, the cross sectional echocardiograms clearly showed that the unruptured right coronary sinus to left ventricle aneurysm would not interfere with the mitral valve mechanism. A very large aneurysm, especially in the presence of atrial fibrillation, might interfere with the opening of the anterior mitral leaflet. Severe aortic regurgitation will certainly cause premature mitral valve closure. Nevertheless, an unruptured aneurysm per se is unlikely to cause mitral regurgitation.

With the advent and wide application of echocardiography, an unruptured aortic sinus aneurysm will be diagnosed at the bedside and no longer be "one of the silent congenital lesions to be suspected when bacterial endocarditis develops in a heart apparently previously healthy."1

\section{References}

1 Jones AM, Langley FA. Aortic sinus aneurysms. $\mathrm{Br}$ Heart f 1949; 11 : 325-41.

2 Sakakibara S, Konno S. Congenital aneurysm of the sinus of Valsalva. Anatomy and classification. Am Heart f 1962; 63: 405-24.

3 Metras D, Coulibaly AO, Ouattara K. Calcified unruptured aneurysm of sinus of Valsalva with complete heart block and aortic regurgitation. Successful repair in one case. Br Heart $\mathcal{F}$ 1982; 48: 507-9. 